\title{
SUITABILITY OF AVERAGING GPS/GNSS PATHS TO BUILD GEOMETRICALLY CORRECT DIGITAL ROAD MAPS
}

\author{
Valerio BAIOCCHI ${ }^{1}$, Domenica COSTANTINO ${ }^{2}$, Felicia VATORE ${ }^{1}$
}

DOI: 10.21163/GT_2017.122.01

\begin{abstract}
:
At present, almost all vehicles have one or more GPS receivers on board. Usually these receivers work in point positioning mode, with deviations of several meters in planimetry and even higher in altitude. Such accuracy is not sufficient for the representation of the road network, both for assisted and automatic navigation purposes and for the creation or updating of large-scale maps. Many free cartographic sources are based on the idea that the continuous repetition of tracking with point positioning receivers makes the measurements converge towards results closer to the actual position of the roads travelled. Here we have experimented whether, by averaging the results of hundreds of tracks found on the same route, we can arrive at accuracy compatible with those needed for navigation and cartography that we have set here compatible with that for the traditional cartography scale 1:5000. The results show that in good conditions of visibility of the sky good results are obtained by averaging a limited number of tracks, more paths are needed in urban areas, while results are not reliable in areas with dense vegetation cover.
\end{abstract}

Key-words: GPS / GNSS, Road maps, Open source, mobile phone, accuracy.

\section{INTRODUCTION}

GPS/GNSS (Global positioning system/ Global navigation satellite systems) has emerged in recent decades as the most widespread positioning technique at global level (Langley et al., 2017). In Differential mode (DGPS), available on professional receivers, the system arrives at centimeter or subcentimetric accuracy allowing applications of high precision including metric verification of the accuracy of satellite images (Pulighe et al., 2016), the georeferencing of of large-scale historical maps (Baglioni et al., 2013), archaeological historical surveys (Ercoli et al., 2016; Costantino et al., 2016), altimetric checks of the DEM (Riguzzi, 2008) or structural monitoring (Dardanelli et al., 2014).

On the other hand, personal GPS receivers in recent decades have had a widespread and global diffusion, practically every mobile phone terminal currently has a GPS/GNSS reception card that allows its location but with accuracy only decametric. The receivers mounted on the mobile terminals, in fact, work almost entirely in single point positioning mode which, after the elimination of select availability (Clinton, 2000) have reached a few meters of planoaltimetric accuracy, before then impossible to achieve for instruments of this class (Satirapod et al., 2001). Since then, many new strategies have been designed to improve accuracy, for example by limiting the effects of multipath, which is a very important effect in urban areas or with plant cover (Pugliano et al., 2016). But the only significant upgrade of single point positioning receivers was the recent introduction of Global Navigation Satellite Systems (GNSS) techniques with the extension to other GNSS

\footnotetext{
${ }^{1}$ Sapienza University of Rome, I-00184, Rome, Italy, valerio.baiocchi@uniroma1.it;

${ }^{2}$ Politecnico di Bari, I-70126 Bari, Italy,domenica.costantino@poliba.it;
} 
constellations (for example for Samsung, Galaxy III and IPhone, from 4s) bringing some improvements (Angrisano et al., 2013). In any case, the geometric accuracy of a trace detected with a receiver in single point positioning is not compatible with the requirement of accuracy for medium to large scale mapping and digital road maps for navigation. To have a representation of the possible approximations, see Fig. 1 where the track recorded by a terminal absolutely stationery for two hours is represented, in a very urbanized area and therefore with poor visibility of the sky. The demand for road maps for road navigation is increasing dramatically, both for personal navigators and for assisted navigation (Seif \& $\mathrm{Hu}, 2016$ ) as well as for the identification of the optimal path (Nicoară \& Haidu, 2014).

Several studies in the literature have been carried out to compare free cartography, the most famous of which is certainly Open street map (OSM). In this direction, original tools and algorithms for the geometric and semantic validation of free maps (Graser et al., 2014; Brovelli et al., 2017) have also been developed. Here, on the other hand, it was interesting to understand, from a purely geometric point of view, whether the average paths converge towards a path with planimetric accuracy compatible with the above mentioned requirements and, if it is possible to estimate after how many repetitions this happens.

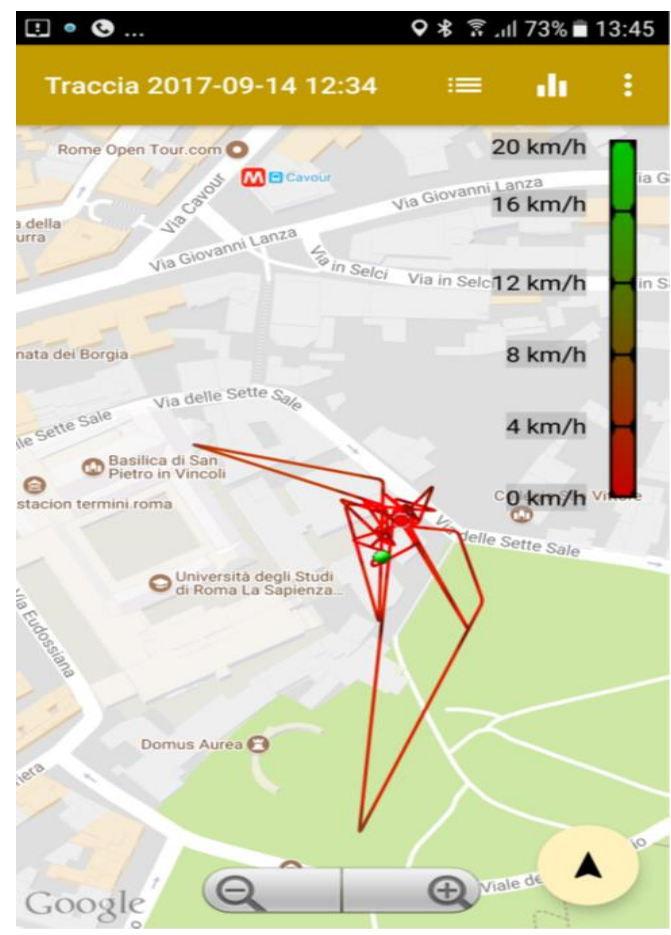

Fig. 1 Track recorded in 2 hours by a terminal absolutely stationary in urban area in the position highlighted by the green dot (the figure is about 220 meters wide).

In literature, the problem of obtaining a track from GPS Single Point Positioning points has been tackled in order to reconstruct the path of a specific vehicle (Gong \& Manoharan, 2011), to identify road junctions (Xie and al., 2017) and also, as in the case under study, to reconstruct an average path from multiple tracks (Brundson, 2017). 


\section{AVAILABLE DATA}

The survey was carried out along a very varied route that starts from the great ring road of Rome (G. R. A.) to reach the city of Latina along the regional road 148 Pontina (SR 148). Most of the routes come back from here, while others reach the A1 motorway at Colleferro, passing through the Lepini mountains.

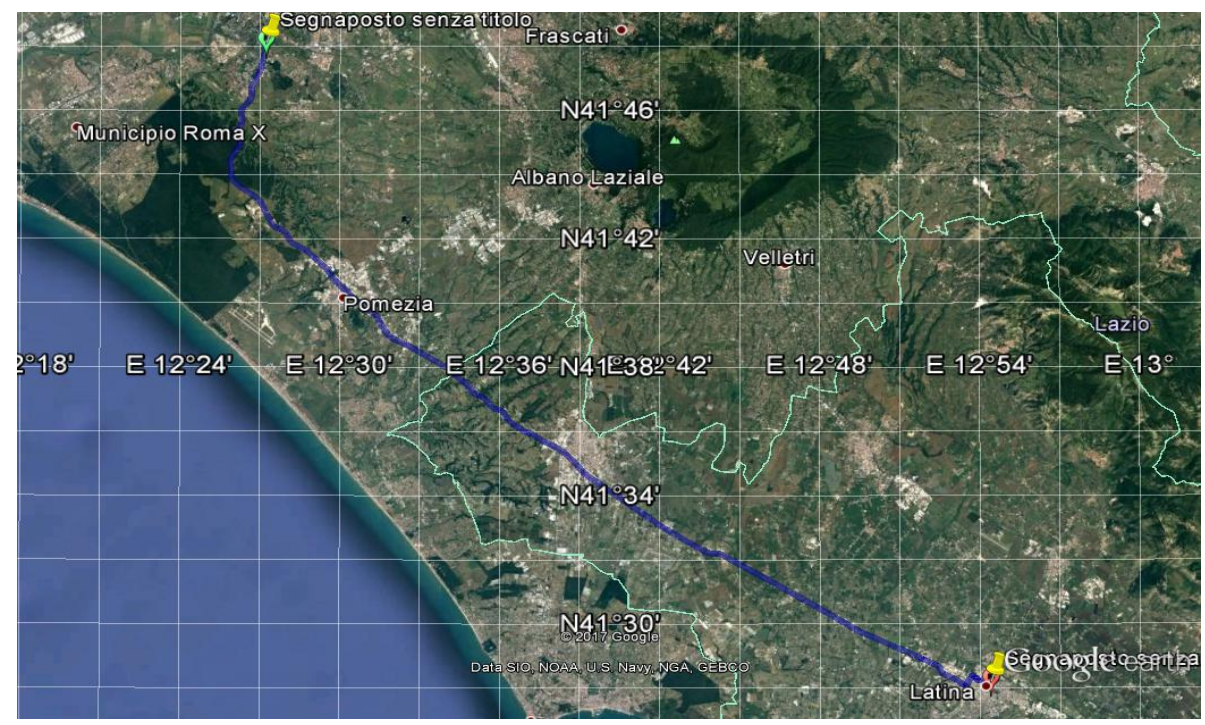

Fig. 2 The first section of the route, the grid spacing is 2' (Lat) *3' (Long), equivalent to approximately: $3702.6 \mathrm{~m}(\mathrm{~N}) * 4142.8 \mathrm{~m}(\mathrm{E})$.

More in detail the first segment (Fig. 2) is practically all on SR 148, separate carriageway road with two lanes per direction plus emergency lane. The maximum permitted speed is $90 \mathrm{~km} / \mathrm{h}$ or $70 \mathrm{~km} / \mathrm{h}$, the routes were run at speeds close to the limits when traffic conditions allowed. This section of the route is often straight both in planimetry and altitude, thanks to the extremely flat nature of the area, the altitude varies very slowly from about 40 meters of the start, up to about 20 meters at the point of arrival. For the first $61.6 \mathrm{~km}$. the route is extra urban, for the remaining $2.8 \mathrm{~km}$. cross the city centre of Latina until you reach the office of the Engineering Faculty in the northeastern part of the city.

The second section (Fig. 3), $48.9 \mathrm{~km}$ long, starts from an urban section and goes from the City of Latina to the motorway toll booth "Colleferro" near the city of the same name, passing through a very articulated area both from the point of view of the planimetric route and for altitude differences. The heights vary from about 20 meters of the start, up to a maximum of about 430 meters at midway to then descend back to about 235 meters of the point of arrival. The road in this section is mainly single carriageway for the two directions of travel, not separated by traffic dividers, therefore the speed limits are between 50 and 90 $\mathrm{Km} / \mathrm{h}$, but often the road conditions suggest even lower speeds. The characteristic that distinguishes this stretch, compared to the other two, is the frequent vegetal cover, partly present only seasonally, and partly present in all seasons of the year. 


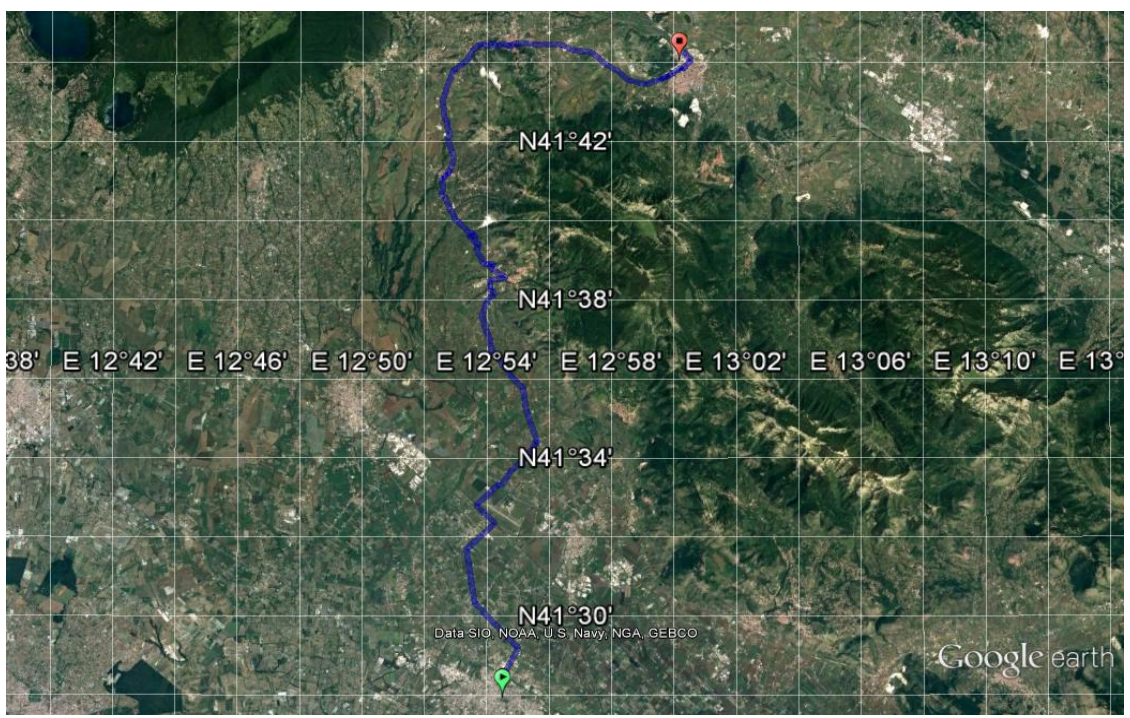

Fig. 3 The second section of the route, the grid spacing is 2' (Lat) *2' (Long), equivalent to approximately: $3702.6 \mathrm{~m}(\mathrm{~N}) * 2761.8 \mathrm{~m}(\mathrm{E})$.

This feature, together with the more mountainous morphology of this section, has limited the visibility of GNSS constellations, influencing the final results of the survey as we will see later.

Finally, the third section (Fig. 4) it runs for $59.5 \mathrm{~km}$. along the Naples-Milan motorway (A1) from Colleferro tollgate to the exit of the same motorway on the G. R. A., the entire section has separate carriageways for the two directions with three lanes in each direction. The route is straight, both planimetrically and altimetrically, with elevations that vary almost constantly, from about 230 from the start to about $50 \mathrm{~m}$. of the arrival point. The speed limit is almost all the way up $130 \mathrm{~km} / \mathrm{h}$. On this route, the reliefs were carried out hundreds of times during different seasons, in particular the entire first section, outward and return journeys were made 173 times, the entire route, including the three stretches, 121 times, plus numerous other reliefs of incomplete routes. In the case of multi-lane carriageways, the one to the right was always occupied, but always where traffic conditions allowed it.

The surveys were carried out on board "C" or "medium cars" segment cars with the cellular terminals installed on the dashboard by means of commercial type supports. The installation on the front of the vehicle, inside the windscreen, exposes the relief to the wellknown effects of Multipath and cycle slip, which is why, in scientific experiments; external antennas are usually used attached to the top of the vehicle (Fastellini et al., 2011). In this experiment the goal was recreating the conditions of acquisition by the user of free databases and therefore the most widespread installation on the dashboard with its defects was used.

Telephone terminals with different reception characteristics of the GNSS constellation have been used, starting from a terminal equipped with a satellite receiver and with which it was therefore necessary to use an external receiver connected via bluetooth, up to terminals of the latest generation with "on board" receivers capable of receiving multi-constellation 
and integrated by other sensors useful for positioning. Details of the GNSS reception characteristics are given in Table 1.

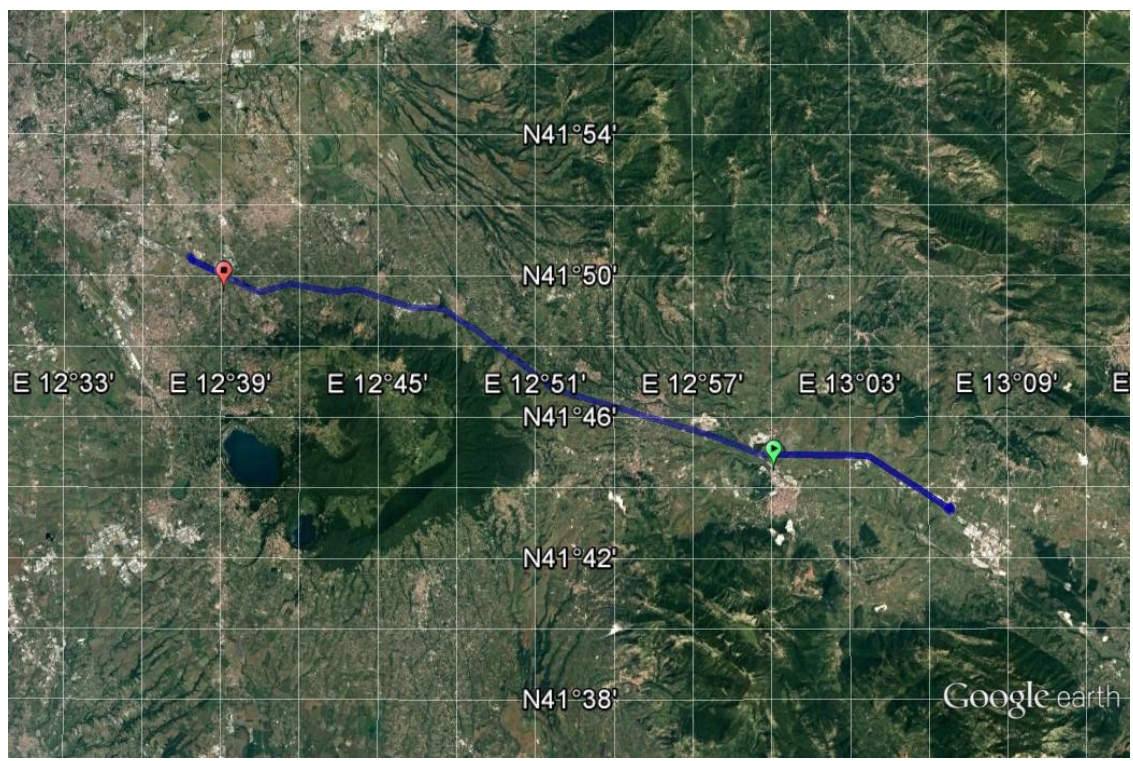

Fig. 4 The third section of the route, the grid spacing is $2^{\prime}$ (Lat) *3' (Long), equivalent to approximately: $3702.6 \mathrm{~m}(\mathrm{~N}) * 4142.8 \mathrm{~m}(\mathrm{E})$.

The GPS receiver antenna has therefore always been in approximately the same position, even though the radio mobile terminals varied with the exception of the previously mentioned first terminal used whose antenna could be installed much closer to the windscreen, increasing the constellation's visibility. The geometric accuracy of the reliefs carried out has been evaluated by comparison with the Regional Technical Cartography (C. T. R.) 1:5000 scale maps, recently (March 2017) freely made available by the Regional Administration of Lazio, both in Raster format (.tif and .pdf) and in vector format (Region Lazio, 2017). The cartography was derived from aerial photogrammetric flights with various update dates, never before 2002.

\section{METHODOLOGY}

The first data processing was the necessary filtering of the outliers; this operation was performed by adopting a simple criterion based on distance and eliminating all the nodes that were not included in a buffer of 20 meters outside the lanes normally practicable. For this purpose, a width of this area was estimated, on a section by section basis, considering 3.75 meters for the width of the transitable lanes and a dimension varying between 1 and 2.5 meters for the traffic partition, where present.

This first filtering has already demonstrated the different results of the routes in relation to the visibility of the GNSS constellation, the percentage of points above the threshold was in fact only $9 \%$ in an open flat area (mainly part of the stretch " 1 " and the entire stretch "3"), while between 13 and $20 \%$ of points were discarded in an urbanized or extra-urban articulated area (mainly in the stretch "2") and up to $87 \%$ in an area. 
Table 1. Used terminal equipment, "N.D." is for "not declared by the manufacturer".

\begin{tabular}{|l|l|l|l|l|l|}
\hline Brand & Model & O.S. & $\begin{array}{l}\text { Receiver } \\
\text { built in }\end{array}$ & Multiconstellation & Other sensors \\
\hline Nokia & E65 & Symbian S60 & No & No: only GPS & No \\
\hline Blackberry & Storm & $\begin{array}{l}\text { RIM } \\
\text { BlackBerry } \\
\text { OS } 4.7\end{array}$ & Yes & N. D. & N.D. \\
\hline Samsung & $\begin{array}{l}\text { Galaxy } \\
\text { ace }\end{array}$ & Android 2.0 & Yes & No: only GPS & N.D. \\
\hline Samsung & $\begin{array}{l}\text { Galaxy III } \\
\text { mini }\end{array}$ & $\begin{array}{l}\text { Android } 4.1 \\
\text { Jelly Bean }\end{array}$ & Yes & $\begin{array}{l}\text { Yes: GPS and } \\
\text { GLONASS }\end{array}$ & $\begin{array}{l}\text { Accelerometer, } \\
\text { Gyroscope, } \\
\text { Compass }\end{array}$ \\
\hline Samsung & $\begin{array}{l}\text { Galaxy } \\
\text { Core LTE }\end{array}$ & $\begin{array}{l}\text { Android } \\
5.0 .2\end{array}$ & Yes & $\begin{array}{l}\text { Yes: GPS, Glonass, } \\
\text { Beidou }\end{array}$ & Accelerometer \\
\hline Samsung & A5 2016 & Android 7.0 & Yes & $\begin{array}{l}\text { Yes: GPS and } \\
\text { Glonass }\end{array}$ & $\begin{array}{l}\text { Accelerometer, } \\
\text { Compass }\end{array}$ \\
\hline
\end{tabular}

Subsequently, all the paths filtered by the outliers were averaged to get a single path as it is done in the open source street maps. The approaches to this type of operation described in the literature are manifold (Morris, 2004), but most are based on the idea of to find a curve running through the' middle' of the point cloud. One way of defining the' middle curve' is to say that it is the curve minimizing the total squared distances (Brundson, 2007) for more details please refer to the literature specified. Here it was decided to use the implementation of this strategy in R proposed by Faber, 2015.

As already mentioned, original tools and algorithms for the geometric and semantic validation of free maps have also been developed in the literature. At this stage, it was only of interest to verify that the planimetric discards were compatible with those of a classic 1:5000 scale cartography, which, in this case, is our comparison. From this point of view, the accuracy that we are asking our tracks for is even greater, as we are comparing them with a cartography already affected by its scraps. It was also decided to verify how many tracks were approximately necessary to make the solution converge towards a path compatible with the limits for cartography 1:5000, which is our reference scale.

Different tolerances are proposed and applied for the planimetric waste, for example some national public administrations (Tuscany Region, 2003) foresees the following constraints: the maximum permissible deviation "tp" in the planimetric position of clearly identifiable points on the map with respect to the same ground points, the position of which has been determined by measurements of sufficient precision, is not greater than:

$$
\begin{aligned}
& \left(\mathrm{dE}^{2}+\mathrm{dN}^{2}\right)^{1 / 2} \mathrm{tp}=1.50 \mathrm{~m} \text { for } 90 \% \text { of the points } \\
& \left(\mathrm{dE}^{2}+\mathrm{dN}^{2}\right)^{1 / 2} \mathrm{tp}=2.50 \mathrm{~m} \text { for } 10 \% \text { of points }
\end{aligned}
$$

To define these ranges, once again buffers have been created at the corresponding distances and the number of points of the paths falling within the buffers relative to the two thresholds has been verified. 
Obviously, the buffers have been differentiated according to the direction of travel that determines the lane used by the vehicle at the time of the survey.

\section{RESULTS AND DISCUSSION}

The first check that was made is whether using all the available tracks would reach the set tolerance values. In this respect, we need to bear in mind two issues with opposite effects. On the one hand, we must always bear in mind that our comparison data (1:5000 map) is affected by an error of its own that leads us to underestimate the accuracy of our tracks.

On the other hand, we are confronted not with the middle line but with the width of the lanes relative to the direction of travel in their totality and therefore with a width of several meters. This causes a certain overestimation of our accuracy because even points not perfectly falling in their correct position can fall into one of the lanes and therefore be considered correct. This last detail is always inherent when we are considering maps for land navigation derived from vehicles in motion on it that cannot always run along the same lane but must adapt to traffic conditions.

On average, therefore, all the already filtered paths of the various segments of our route, it has been verified that in the two open and flat areas, namely the "1" route for the extra-urban part and the "3" route in its totality, we have a total achievement of the established tolerance limits, in fact, $93 \%$ of the points have returned in the buffer of $1.50 \mathrm{~m}$ while $7 \%$ have returned in the buffer of $2.50 \mathrm{~m}$.

In mountainous areas (mainly the central part of route 2) and in urbanized areas, similar results were still recorded, reaching the expected values in most of the route $(82 \%$ and $84 \%$ respectively). In areas with dense plant cover (trace 2) the nodes are only tolerated in $12 \%$ of the trace. Subsequently, it was decided to evaluate after how many tracks the necessary accuracy was reached, in order to have a reference value of the necessary repetitions.

For straight segments, it has been verified that it is already within the optimum values with 32 routes in the first sector and after 27 in the third section, this difference is probably due to the greater width of the motorway section that characterizes the third section of the route. As already mentioned, in fact, the greater width of the road infrastructure and its appliances increases the area in which the points detected by the GPS/GNSS Receiver can positively fall.

The same test was carried out in urban areas and in the very articulated zones, achieving the achievement of tolerances after 81 and 67 paths respectively. Obviously, these tests were only carried out in the sections that had previously reported results within the established tolerances, outside of which it would not make sense to do the same tests. For the same reason, this test was not carried out in areas with high tree coverage.

\section{CONCLUSIONS}

From the experiments carried out, it has been verified that averaging the results of numerous GPS/GNSS tracks acquired in point positioning mode, it is possible to obtain tracks with greater accuracy and such as to be within the tolerances necessary for the construction and updating of digital road maps. No significant improvements were observed using more GNSS constellations instead of GPS alone. These results are 
particularly interesting in open areas where even with a few dozen repetitions (about 30) reliability can be achieved that are compatible with the expected accuracy.

In the most articulated and urbanized areas, a reliable repetition value can be about 90 , while in areas with dense plant cover and under tunnels and viaducts the results do not seem to converge reliably even after hundreds of repetitions.

This latter result, easily predictable before experimentation, highlights the nonapplicability of this methodology in such situations, in which it must be remembered; however, not even the classic photogrammetric methods obtain acceptable results.

Further surveys are still in progress to evaluate if also in urban areas it's possible to reach $100 \%$ of the tolerance value repeating more and more time the same roads.

\section{ACKNOWLEDGMENT}

This paper was developed with the financial support of Education and University (MIUR) mainly with the PRIN Project no. 2008ZKJSJ2_004 and with other grants.

\section{R E F E R E N C E S}

Angrisano, A., Gaglione, S., Gioia, C. (2013) Performance assessment of GPS/GLONASS single point positioning in an urban environment. Acta Geodaetica et Geophysica, 48(2), pp. 149-161.

Baglioni, R., Baiocchi, V., Dominici, D., Milone, M.V., Mormile, M. (2013) Historic cartography of L'Aquila city as a support to the study of earthquake damaged buildings. Geographia Technica, 8(1), pp. 1-9.

Brovelli, M. A., Minghini, M., Molinari, M. and Mooney, P. (2017) Towards an Automated Comparison of OpenStreetMap with Authoritative Road Datasets. Transactions in GIS, 21(2), pp. 191-206.

Clinton, B. (2000) White house's web page: http://www.pub.whitehouse.gov/urires/I2R?urn:pdi://oma.eop.gov.us/2000/5/2/8.text.2

Costantino, D., Angelini, M.G., Baiocchi, V. (2016) Integrated surveying for the archaeological documentation of a neolithic site. Geographia Technica, 11(2), pp. 39-49.

Dardanelli, G., La Loggia, G., Perfetti, N., Capodici, F., Puccio, L., Maltese, A. (2014) Monitoring displacements of an earthen dam using GNSS and remote sensing. Proc. SPIE 9239, Remote Sensing for Agriculture, Ecosystems, and Hydrology, XVI, 923928 (21 October 2014). DOI: 10.1117/12.2071222.

Ercoli, M., Brigante, R., Radicioni, F., Pauselli, C., Mazzocca, M., Centi, G., Stoppini, A. (2016) Inside the polygonal walls of Amelia (Central Italy): A multidisciplinary data integration, encompassing geodetic monitoring and geophysical prospections. Journal of Applied Geophysics, 127, pp. 31-44.

Faber, M. (2005) R (programming language) / Average tracks [Online] http://wiki.openstreetmap.org/wiki/R_ (programming_language) / Average_tracks [Accessed August 2017].

Fastellini, G., Radicioni, F., Stoppini, A. (2011) Field tests on GNSS and inertial systems for transport fleet monitoring in urban environment. Italian Journal of Remote Sensing / Rivista Italiana di Telerilevamento, $\mathbf{4 3}(2)$, pp. 41-54.

Gong, X. \& Manoharan S. (2011) On predicting vehicle tracks. Proceedings of 2011 IEEE Pacific Rim Conference on Communications, Computers and Signal Processing, Victoria, BC, pp. 3136.

Graser, A., Straub, M., Dragaschnig, M. (2014) Towards an open source analysis toolbox for street network comparison: Indicators, tools and results of a comparison of osm and the official Austrian reference graph. Transactions in GIS, 18(4), pp. 510-526. 
Langley R. B., Teunissen P.J., Montenbruck O. (2017) Introduction to GNSS. In: Teunissen P.J., Montenbruck O. (eds.) Springer Handbook of Global Navigation Satellite Systems. Springer, Cham.

Nicoară P-S. \& Haidu I. (2014) A GIS based network analysis for the identification of shortest route access to emergency medical facilities. Geographia Technica, 9(2), pp. 60-67.

Pugliano, G., Robustelli, U., Rossi, F., Santamaria, R. (2016) A new method for specular and diffuse pseudorange multipath error extraction using wavelet analysis. GPS Solutions, 20(3), pp. 499508.

Pulighe, G., Baiocchi, V., Lupia, F. (2016) Horizontal accuracy assessment of very high resolution Google Earth images in the city of Rome, Italy. International Journal of Digital Earth, 9(4), pp. 342-362.

Satirapod, C., Rizos, C.,Wang J. (2001) GPS single point positioning with SA off: how accurate can we get? Survey Review, 36(282), pp. 255-262.

Seif, H., Hu, X. (2016) Autonomous Driving in the iCity-HD Maps as a Key Challenge of the Automotive Industry. Engineering, 2(2), pp. 159-162.

Xie, X., Liao, W., Aghajan, H., Veelaert, P., Philips, W. (2017) Detecting Road Intersections from GPS Traces Using Longest Common Subsequence Algorithm. ISPRS Int. J. Geo-Inf., 6(1).

http://cartografia.regione.lazio.it/cartanet/catalogo/catalog?folderinside=ortofoto\#.Wb0Q57JJYy4

[Online] [Accessed August 2017].

www.apple.com [Online] [Accessed August 2017].

www.blackberry.com [Online] [Accessed August 2017].

www.nokia.com [Online] [Accessed August 2017].

www.samsung.com [Online] [Accessed August 2017]. 